\title{
Double-logarithmic asymptotics of the electromagnetic form factors of the electron and quark
}

\author{
B.I. Ermolaev \\ A.F. Ioffe Physico-Technical Institute, 194021 St.Petersburg, Russia \\ and \\ S.I. Troyan \\ St.Petersburg Institute of Nuclear Physics, 188350 St.Petersburg, Gatchina, Russia
}

\begin{abstract}
The asymptotic behaviour of the electromagnetic form factors of the electron and quark is obtained in the double-logarithmic approximation for Sudakov kinematics, i.e. for the case that the value of the momentum transfer is much greater than the mass of the particle.
\end{abstract}

PACS numbers: 12.38.Cy

\section{INTRODUCTION}

The interaction of an electron or quark with an electromagnetic field is described in terms of two independent form factors $f$ and $g$ :

$$
\Gamma_{\mu}=\bar{u}\left(p_{2}\right)\left[\gamma_{\mu} f\left(q^{2}\right)-\frac{\sigma_{\mu \nu} q_{\nu}}{2 m} g\left(q^{2}\right)\right] u\left(p_{1}\right)
$$

where $\sigma_{\mu \nu}=\left[\gamma_{\mu} \gamma_{\nu}-\gamma_{\nu} \gamma_{\mu}\right] / 2, q=p_{2}-p_{1}$ is the momentum transferred to the electron or the quark, $m$ is the electron or quark mass and both the form factors $f$ and $g$ depend on $q^{2}$.

In the Born approximation $f=1$ and $g=0$. The calculation of one-loop radiative corrections [1] shows that the form factor $f$ depends on the infrared cutoff as well as on the ultraviolet one. In contrast, the form factor $g$ in the one-loop approximation is both ultraviolet- and infrared- stable. As the form factor $g$ contributes to the value of the anomalous magnetic moment, it has been calculated with great accuracy by direct graph-by-graph calculations in the case that $q^{2}=0$. The most recent review of such results is given in [2]. Meanwhile, the form factor $f$ was calculated many years ago in the "opposite" kinematical region of very large momentum transfer:

$$
-q^{2} \gg m^{2}
$$

in the leading logarithmic approximation (LLA), where the most important, double logarithmic contributions to all orders in the QED coupling $\alpha$ of the electron are taken into account. The sum of such contributions, the double logarithmic (DL) asymptotic behaviour of the form factor of the electron in the kinematical region (2), is

$$
f=\exp \left[-\frac{\alpha}{4 \pi} \ln ^{2}\left(\frac{-q^{2}}{m^{2}}\right)\right]
$$

The famous expression (3), obtained by V.V. Sudakov [3], was actually the birth of the approach that is so popular at present - the double logarithmic approximation (DLA) - where only the leading contributions $\sim\left(\alpha \ln ^{2}\left(-q^{2}\right)\right)^{n}$ are taken into account to all orders of the perturbation series. The generalization of the Sudakov form factor of Eq. (3) to the quarks of QCD obtained in [4] amounts to replacing $\alpha$ by $\alpha_{s} C_{F}$ in Eq. (3), where $C_{F}=\left(N^{2}-1\right) / 2 N=4 / 3$ for the colour group $S U(3)$.

The exponential fall in Eq. (3) as $-q^{2}$ increases corresponds to a suppression of the non-radiative hard scattering of an electron by a virtual photon. The amplitude taking into account the bremsstrahlung of $n$ "soft" photons was shown in [5] to be a product of independent factors :

$$
f_{n}=B_{1} B_{2} \ldots B_{n} f\left(q^{2}\right),
$$

where the bremsstrahlung factors $B_{i}$ are given by (we drop the QED coupling here)

$$
B_{i}=\frac{p_{2} l_{i}}{p_{2} k_{i}}-\frac{p_{1} l_{i}}{p_{1} k_{i}}
$$

$l_{i}$ being the polarization vector and $k_{i}$ the momentum of the $i$-th emitted photon $(i=1, \ldots, n)$. As $f$ in Eq. (5) does not depend on $k_{i}$, and each of $B_{i}$ does not depend on $k_{j}$ with $j \neq i$, Eq. (5) leads to a Poisson energy spectrum 
for bremsstrahlung photons in the DLA. The violation of the Poisson distribution in QCD for the emission of "soft" gluons in the DLA was obtained in [6] by calculating Feynman graphs up to order $\alpha_{s}^{2}$. The generalization of the form factor $f$ in Eq. (4) to QCD was given in [7], [8].

As follows from the lowest, one-loop approximation [1], the form factor $g$ of the electron in the kinematic region (2),

$$
g^{(1)}\left(q^{2}\right)=-\frac{m^{2}}{q^{2}} \frac{\alpha}{\pi} \ln \left(\frac{-q^{2}}{m^{2}}\right),
$$

compared to the form factor $f$, lacks one large logarithmic factor and is suppressed by the power factor $m^{2} / q^{2}$. However, in spin-flip scattering amplitudes the relatively large form factor $f$ is multiplied by the same small factor $m^{2} / q^{2}$ and the effects of the form factor $g$ become sizeable.

In the present work we calculate the form factor $g$ for the electron and the quark in the kinematical region (2) in the double logarithmic approximation (DLA). Then, using the results of [8], we obtain relations between the radiative (inelastic) electromagnetic form factors of the electron and quark.

The paper is organized as follows: in Section 2 we prove the factorization property for the case of the elastic form factor $g$ of the electron and calculate $g$ in DLA first by direct calculation of Feynman graphs, then by constructing and solving infrared evolution equations $f$ and $g$ obey. In Section 3 we generalize that result to QCD. In Section 4 we show how the previous results can be generalized to the case of the inelastic form factors of the electron and quark. Section 5 is devoted to concluding remarks.

\section{ELASTIC FORM FACTORS OF THE ELECTRON}

In the lowest, one-loop approximation $f$ and $g$ form factors of electron were calculated long ago in [1]. In the Feynman gauge the only graph yielding the logarithmic contributions to $f$ and $g$ in the kinematics (2) is shown in Fig. 1. The result is 11

$$
g^{(1)}\left(q^{2}\right)=-\frac{m^{2}}{q^{2}} \frac{\alpha}{\pi} \ln \left(\frac{-q^{2}}{m^{2}}\right)
$$

In order to reproduce (6) in the manner easy to generalize to higher loops with the DL accuracy, we use the Sudakov parameterisation [3]. According to it, momentum $k_{i}$ of each soft virtual particle (a virtual photon) is expressed through the longitudinal variables $\alpha_{i}, \beta_{i}$ and the transverse momentum $k_{i \perp}$ :

$$
k_{i}=\alpha_{i} p_{2}^{\prime}+\beta_{i} p_{1}^{\prime}+k_{i \perp}
$$

where $\left(k_{i \perp} p_{1}\right)=\left(k_{i \perp} p_{2}\right)=0, s \equiv 2\left(p_{1} p_{2}\right) \approx-q^{2}$ and $p_{1}, p_{2}$ are the momenta of the electron (see Fig. 11). Then,

$$
p_{1}^{\prime}=p_{1}-\left(m^{2} / s\right) p_{2}, \quad p_{2}^{\prime}=p_{2}-\left(m^{2} / s\right) p_{1}
$$

so that $\left(p_{1}^{\prime}\right)^{2}=\left(p_{2}^{\prime}\right)^{2}=0$.

Applying the Feynman rules to the graph in Fig. 1, we obtain the expression

$$
\begin{array}{r}
\Gamma_{1}=-\imath\left(\alpha / 4 \pi^{3}\right) \int d k_{1} \bar{u}\left(p_{2}\right) \gamma_{\nu} \frac{\left(\hat{p}_{2}+m-\hat{k}_{1}\right)}{\left[\left(p_{2}-k_{1}\right)^{2}-m^{2}+\imath \epsilon\right]} \\
\gamma_{\mu} \frac{\left(\hat{p}_{1}+m-\hat{k}_{1}\right)}{\left[\left(p_{1}-k_{1}\right)^{2}-m^{2}+\imath \epsilon\right]} \gamma_{\nu} u\left(p_{1}\right) \frac{1}{\left[k^{2}+\imath \epsilon\right]} .
\end{array}
$$

Simplifying the spinor structure in Eq. (10) and integrating it over $k_{1 \perp}$ with the replacement 1

$$
\frac{1}{k_{1}^{2}+\imath \epsilon} \rightarrow-2 \imath \pi \delta\left(s \alpha \beta-k_{1 \perp}^{2}\right)
$$

\footnotetext{
${ }^{1}$ This replacement is correct for calculations in double-logarithmic and single-logarithmic approximations. One can prove it calculating the integral of Eq. (10) by taking residues.
} 
so that

$$
\int d k_{1} \frac{F\left(k_{1}\right)}{k_{1}^{2}+\imath \epsilon} \approx-2 \imath \pi \int_{0}^{1} d \alpha_{1} \int_{0}^{1} d \beta_{1} F\left(\alpha_{1}, \beta_{1}\right) \Theta\left(s \alpha_{1} \beta_{1}-\mu\right),
$$

with $\mu$ being an infrared cutoff defined as

$$
k_{1 \perp}>\mu \geq m
$$

we arrive at

$$
\begin{aligned}
\Gamma_{1}= & -\left(\gamma_{\mu}\right) \frac{\alpha}{2 \pi} \int \frac{d \alpha_{1}}{\left(\alpha_{1}-\left(m^{2} / s\right) \beta_{1}\right)} \frac{d \beta_{1}}{\left(\beta_{1}-\left(m^{2} / s\right) \alpha_{1}\right)} \Theta\left(s \alpha_{1} \beta_{1}-\mu^{2}\right)+ \\
& \frac{\alpha}{4 \pi} 2 m\left(\sigma_{\mu \nu} q_{\nu}\right) \int \frac{d \alpha_{1}}{\left(\alpha_{1}-\left(m^{2} / s\right) \beta_{1}\right)} \frac{d \beta_{1}}{\left(\beta_{1}-\left(m^{2} / s\right) \alpha_{1}\right)}\left[\alpha_{1}\left(1-\alpha_{1}\right)+\beta_{1}\left(1-\beta_{1}\right)\right] \Theta\left(s \alpha_{1} \beta_{1}-\mu^{2}\right) .
\end{aligned}
$$

We have used in Eq. (14) the notations $\left(\gamma_{\mu}\right) \equiv \bar{u}\left(p_{2}\right) \gamma_{\mu} u\left(p_{1}\right),\left(\sigma_{\mu \nu} q_{\nu}\right) \equiv(1 / 2) \bar{u}\left(p_{2}\right) \sigma_{\mu \nu} q_{\nu} u\left(p_{1}\right)$.

Let us comment on Eq.(14). We retained only leading contributions to the form factors $f$ and $g$ in Eq.(14). The leading contribution to $f$ is the double logarithmic one, with one logarithm as a result of integrating over $\alpha_{1}$ and the other logarithm over $\beta_{1}$. As the denominator in Eq.(14) is, in essence, just $\alpha_{1} \beta_{1}$, obtaining the two logarithms in the leading contribution to $f$ corresponds to dropping all the Sudakov variables in the numerator of Eq.(14). In order to get the leading contribution to $g$ one must not neglect $\alpha_{1}, \beta_{1}$ in the numerator. Keeping either $\alpha_{1}$ or $\beta_{1}$ in the numerators of Eq. (14) immediately kills one logarithmic contribution and keeping products $\alpha_{1} \beta_{1}$ kills the both logarithms. In the present paper we discuss only the leading contribution to $g$, therefore through the paper we neglect contributions containing products of $\alpha_{i}$ and $\beta_{i}$ (i $\left.=1,2, ..\right)$. In particular, it means that we neglect contributions containing powers of $k_{\perp}$ because $k_{1}^{2}=s \alpha_{1} \beta_{1}$. However, we account for the terms in the numerator of Eq. (14) containing powers of $\alpha_{1}$ and of $\beta_{1}$ separately. Then, as $m$ is the small parameter in the kinematics (2), we neglected in the numerators of Eq.(14) contributions of order $m$ to the form factor $f$ and contributions of order $m^{2}$ to $g$. We will do the same neglections when we consider the higher loop contributions to those form factors.

Comparing Eq. (14) and (11), we see that the contribution of $\Gamma_{1}$ to the form factor $f$ is

$$
-\frac{\alpha}{2 \pi}\left(\gamma_{\mu}\right) \int \frac{d \alpha_{1}}{\left[\alpha_{1}-\left(m^{2} / s\right) \beta_{1}\right]} \frac{d \beta_{1}}{\left[\beta-\left(m^{2} / s\right) \alpha_{1}\right]} .
$$

The DL region of integration in Eqs. (14) and (15) is

$$
1>\alpha_{1}>\left(m^{2} / s\right), \quad 1>\beta_{1}>\left(m^{2} / s\right), \quad \alpha_{1} \beta_{1}>\mu^{2} / s .
$$

Obviously, the integral in Eq. (15) diverges when $\mu=0$, so the cut-off $\mu$ has to be kept . As within DL accuracy one can choose an arbitrary infrared cut-off, we choose, for the sake of simplicity,

$$
\mu=m \text {. }
$$

After that we immediately reproduce the well known one-loop result $f^{(1)}$ for the form factor $f$ with the DL accuracy:

$$
f^{(1)}=-(\alpha / 4 \pi) \ln ^{2}\left(-q^{2} / m^{2}\right)
$$

In the contrast to $f$-form factor, the contribution to form factor $g$ given by the second integral in Eq. (14), is infrared stable. Indeed, using the symmetry in $\alpha_{1}, \beta_{1}$ we rewrite the second integral in Eq. (14) with the logarithmic accuracy as

$$
\int_{0}^{1} \frac{d \beta_{1}}{\beta_{1}} 2 \beta_{1}\left(1-\beta_{1}\right) \int_{m^{2} / s}^{1} \frac{d \alpha_{1}}{\alpha_{1}}=\ln \left(s / m^{2}\right)
$$

Combining the result with the remaining numerical factors in Eq. (14) and using the definition (1) of form factor $g$ we reproduce Eq. (6).

Now let us consider the DL radiative corrections to the one-loop results (18), (6) for $f$ and $g$ coming from an arbitrary $\alpha^{n}$ - order graph. In the Feynman gauge that we use through the present paper, graphs yielding the DL contributions are obtained from the one-loop graph in Fig. 1 by adding to it the photon propagators connecting the lines with momenta $p_{1}$ and $p_{2}$ in an arbitrary way. However, such graphs can not have the closed fermion loops 
because the loops yield the single-logarithmic contribution only. We find it convenient to regard the virtual photon propagators connecting the electron lines with momenta $p_{1}$ and $p_{2}$ as the ones beginning on the line with $p_{1}$ and ending on the line with $p_{2}$. Without loss of generality, we can numerate with numbers $1,2, \ldots, n$ the virtual photons emitted from $p_{1}$-line so that the propagator of the photon with momentum $k_{1}$ begins at the bottom (see Fig. 2). However, basically those emitted photons do not arrive at the $p_{2}$ - line in the same order. For an arbitrary graph, the order for the $p_{2}$ - line is $j_{1}, j_{2}, \ldots, j_{n}$ (see Fig. 2). Thus, any of the graphs contributing to $\Gamma_{\mu}$ correspond to a certain permutation $j_{n}, \ldots, j_{1}$ of the initial order $n, \ldots, 1$. In particular, the permutation $j_{n}, \ldots, j_{1}$ with $j_{1}=1, j_{2}=2, . ., j_{n}=n$ corresponds the ladder graph and the other, non-ladder graphs are obtained from it by permutations of numbers $(n, n-1, \ldots, 1)$ on the $p_{2}$-line. So, in order $\alpha^{n}$ there are $n$ ! different graphs yielding the DL contributions. These graphs yield equal contributions to the form factor $f$ when the electron is off-shell but for the on-shell electron it does not hold.

Again without loss of generality, we assume that the photon with number $j_{n}$ on the $p_{2}$-line has number $r$ on line $p_{1}$ and the photon with number $n$ on line $p_{1}$ has number $j_{r}$ on line $p_{2}$ as it shown in Fig. 2. It leads to the matching

$$
k_{j_{n}}=k_{r}, \quad k_{j_{r}}=k_{n}
$$

We do not need to do matchings between other emitted and absorbed photons, with non-largest Sudakov variables, so we let them be arbitrary. We demonstrate it in Fig. 2 2 by leaving disconnected those photon lines.

Straightforward applying the Feynman rules to the graph in Fig. 22 yields

$$
\begin{aligned}
\Gamma_{n}= & (-\imath)^{n} \frac{\alpha^{n}}{(4 \pi)^{3 n}} \int d k_{1} \ldots d k_{n} \bar{u}_{2} \gamma_{\lambda_{j_{1}}} \frac{\left(\hat{p}_{2}+m-\hat{k}_{j_{1}}\right)}{\left[\left(p_{2}-k_{j_{n}}\right)^{2}-m^{2}\right]} \ldots \gamma_{\lambda_{j_{1}}} \\
& \frac{\left(\hat{p}_{2}+m-\hat{k}_{j_{n}}-\ldots-\hat{k}_{j_{1}}\right)}{\left[\left(p_{2}-k_{j_{1}}-\ldots-k_{j_{n}}\right)^{2}-m^{2}\right]} \gamma_{\mu} \frac{\left(\hat{p}_{1}+m-\hat{k}_{1}-\ldots-\hat{k}_{n}\right)}{\left[\left(p_{1}-k_{1}-\ldots-k_{n}\right)^{2}-m^{2}\right]} \gamma_{\lambda_{n}} \\
& \ldots \frac{\left(\hat{p}_{1}+m-\hat{k}-n\right)}{\left[\left(p_{1}-k_{1}\right)^{2}-m^{2}\right]} \gamma_{\lambda_{1}} u_{1} \prod_{j=0}^{j=n} \frac{1}{k_{j}^{2}} .
\end{aligned}
$$

In terms of the Sudakov variables

$$
\prod d k_{i}=(s / 2)^{n} \prod d \alpha_{i} d \beta_{i} d^{2} k_{i \perp}
$$

In (22) $i$ also runs from 1 to $n$. Integration over every transverse momentum $k_{i \perp}$ can be done with replacement $1 /\left[k^{2}+\imath \epsilon\right]$ by $-\imath 2 \pi \delta\left(k_{i}^{2}\right)$ just like it was done for the one-loop integration (14). After that the integrand in Eq. (21) depends on $\alpha_{i}, \beta_{i}$. In the DLA we must keep all the Sudakov variables $\alpha_{j}, \beta_{j},(j=1, \ldots, n)$ in the denominators of Eq. (21). It is possible only if they obey to the following ordering:

$$
\begin{array}{r}
\alpha_{1}<\alpha_{2}<\ldots<\alpha_{n-1}<\alpha_{1}<1, \\
\beta_{j_{1}}<\beta_{j_{2}}<\ldots<\beta_{j_{n}}<1 .
\end{array}
$$

Eq. (23) reads that all $\alpha_{i}, \beta_{i}$ are small and therefore we can drop all $k_{i}$ in the numerators in (21) compared to $p_{1}$, $p_{2}$ and at the same time keep only linear in $\alpha_{i}, \beta_{j}$ contributions in the denominator. When we do it, we arrive at the double- logarithmic $\alpha^{n}$-th order contribution to the form factor $f$ :

$$
f^{(n)}=(-1)^{n}\left(\frac{\alpha}{2 \pi}\right)^{n} \int \prod \frac{d \alpha_{i} d \beta_{i}}{\alpha_{i} \beta_{i}} \Theta\left(s \alpha_{i} \beta_{i}-\mu^{2}\right) .
$$

However, contributions to $g$ come from the terms in the numerator of Eq. (21) containing the Sudakov variables. Due to the ordering (23), the leading contributions of the numerator of (21) to $g$ come from the biggest of them, depending on $\alpha_{n}$ and on $\beta_{j_{n}}$. So, we keep only such contributions and neglect the others. For the self-consistency of the approach, we can approximate the denominators in (21) by the contributions linear in $\alpha_{i}, \beta_{i}$ for $f$ but we must keep all the powers of $\alpha_{n}$ and $\beta_{j_{n}}$ for contributions to $g$. At the same moment we can use linear approximation for other $\alpha_{i}, \beta_{i}$ with $i \neq n, j_{n}$. So, in order to get the leading contribution to $g$, we approximate the numerator in (21) (cf. (14) ) by

$$
(-2 m)\left(\sigma_{\mu \nu} q_{\mu}\right)(2 s)^{(n-1)}\left[\beta_{j_{n}}\left(1-\beta_{j_{n}}\right)^{(n+1-r)}+\alpha_{n}\left(1-\alpha_{n}\right)^{\left(n+1-j_{n}\right)}\right],
$$

and the denominator, correspondingly, by 


$$
\alpha_{n}\left(1-\alpha_{n}\right)^{\left(n-j_{n}\right)} \beta_{j_{n}}\left(1-\beta_{j_{n}}\right)^{(n-r)} \prod^{\prime} \alpha_{i} \beta_{i} .
$$

We have used the notation $\prod^{\prime}$ to show that this product does not include $\beta_{j_{n}}$ and $\alpha_{n}$. We have neglected in Eq. (25), just like we did for Eq.(14), contributions containing products of $\alpha_{n}$ and $\beta_{j_{n}}$ as well as terms containing $k_{i \perp}, \quad(\mathrm{i}=1,2, \ldots, \mathrm{n})$ because accounting for them leads to loss more than one logarithmic contribution. Combining Eqs. (21), 222, (26) and (25), we obtain $\alpha^{n}$-order contributions of the graph in Fig. 2 to $g$ :

$$
\begin{aligned}
g^{(n)}= & -(-1)^{n}\left(\frac{\alpha}{2 \pi}\right)^{n} 2 \int d \beta_{j_{n}} d \alpha_{n} \frac{\left[\beta_{j_{n}}\left(1-\beta_{j_{n}}\right)+\alpha_{n}\left(1-\alpha_{n}\right)\right]}{\beta_{j_{n}} \alpha_{n}} \int \frac{d \beta_{n} d \alpha_{r}}{\beta_{n} \alpha_{r}} \Theta\left(s \alpha_{r} \beta_{r}-\mu^{2}\right) \\
& \Theta\left(s \alpha_{n} \beta_{n}-\mu^{2}\right) \prod^{\prime} \int \frac{d \alpha_{i} d \beta_{i}}{\alpha_{i} \beta_{i}} \Theta\left(s \alpha_{i} \beta_{i}-\mu^{2}\right)
\end{aligned}
$$

Using the symmetry of Eq.(27) in replacing $\alpha_{n} \rightleftharpoons \beta_{j_{n}}, \alpha_{n} \rightleftharpoons \beta_{j_{n}}$, we rewrite Eq. (27) as

$$
\begin{aligned}
g^{(n)}= & -(-1)^{n}\left(\frac{\alpha}{2 \pi}\right)^{n} 2 \int d \beta_{j_{n}} 2\left(1-\beta_{j_{n}}\right) \int \frac{d \alpha_{j_{n}}}{\alpha_{j_{n}}} \Theta\left(s \alpha_{j_{n}} \beta_{j_{n}}-\mu^{2}\right) \\
& \int \frac{d \beta_{n} d \alpha_{r}}{\beta_{n} \alpha_{r}} \prod^{\prime} \int \frac{d \alpha_{i} d \beta_{i}}{\alpha_{i} \beta_{i}} \Theta\left(s \alpha_{i} \beta_{i}-\mu^{2}\right)
\end{aligned}
$$

The integration regions in Eqs.(28), (24) are identical. They are defined by the ordering (23) and by the $\Theta$-functions. Thus, the only difference between the integrals in Eqs.(28),(24) is the integration over $\beta_{r}$. The integration region over $\alpha_{i},(i=1, . ., n)$ is

$$
\frac{\mu^{2}}{s} \beta_{i}<\alpha_{i}<\alpha_{i+1}
$$

where the upper limit for intergrating over $\alpha_{n}$ is 1 . The region (29) is the same for any graph contributing to $f, g$. Integration over $\beta_{j_{l}}$ runs from $\mu / 2 / s$ to $\beta_{j_{l+1}}$, with $\beta_{j_{n+1}}=1$. It leads to

$$
f^{(n)}=(-1)^{n}\left(\frac{\alpha}{2 \pi}\right)^{n} \int_{\lambda}^{1} \frac{d \beta_{j_{n}}}{\beta_{j_{n}}} F^{(n)}\left(\beta_{j_{n}}, \lambda\right)
$$

where

$$
F^{(n)}\left(\beta_{j_{n}}, \lambda\right)=F^{(n)}\left(\beta_{j_{n}} / \lambda\right)=\int_{\lambda}^{1} \frac{d \beta_{j_{n-1}}}{\beta_{j_{n-1}}} \ldots \int_{\lambda}^{1} \frac{d \beta_{j_{1}}}{\beta_{j_{1}}} \int_{\lambda / \beta_{n}}^{1} \frac{d \alpha_{n}}{\alpha_{n}} \ldots \int_{\lambda / \beta_{1}}^{\alpha_{2}} \frac{d \alpha_{1}}{\alpha_{1}}
$$

and $\lambda=\mu^{2} / s$.

We have arranged the order of integrations in (30),(31) so that Eq. (31) looked similar to Eq. (27) for the contribution to $g^{(n)}$ where only the last integration over $\beta_{j_{n}}$ does not yield a logarithmic contribution whereas each integration in $F$ yields it (see(31)).

Now, using (31), we can express $g^{(n)}$ given by Eq. (28) in terms of $F$ :

$$
g^{(n)}=-(-1)^{n} 2\left(\frac{\alpha}{2 \pi}\right)^{n} \int_{\lambda}^{1} d \beta_{j_{n}} 2\left(1-\beta_{j_{n}}\right) F^{(n)}\left(\beta_{j_{n}} / \lambda\right) .
$$

Replacing $\beta_{j_{n}}$ in Eqs. (30), (32), by $\beta$, we rewrite them as

$$
f^{(n)}=(-1)^{n}\left(\frac{\alpha}{2 \pi}\right)^{n} U_{f}^{(n)}
$$

where

$$
U_{f}^{(n)}(\lambda)=\int_{\lambda}^{1} \frac{d \beta}{\beta} F^{(n)}(\beta / \lambda)
$$

and

$$
g^{(n)}=-(-1)^{n} 2\left(\frac{\alpha}{2 \pi}\right)^{n} \frac{m^{2}}{s} U_{g}^{(n)}
$$


with

$$
U_{g}^{(n)}=\int_{\lambda}^{1} d \beta 2 \beta(1-\beta) F^{(n)}(\beta / \lambda) .
$$

In the DLA,

$$
\int_{\lambda}^{1} d \beta 2 \beta(1-\beta) \ln ^{n}(\beta / \lambda)=\ln ^{n}(1 / \lambda) .
$$

Therefore

$$
U_{g}^{(n)} \approx F^{(n)}(1 / \lambda)
$$

On the other hand, Eq. (34) reads that

$$
\frac{\partial U_{f}^{(n)}}{\partial \ln (1 / \lambda)}=F^{(n)}(\ln (1 / \lambda)) .
$$

Combining Eqs. (38) and (39), we conclude that in order $\alpha^{n}$

$$
U_{g}^{(n)}=\frac{\partial U_{f}^{(n)}}{\partial \ln (1 / \lambda)} .
$$
for :

Summing up (41) over $n$ leads to the following obvious relation between $U_{f}$ and $U_{g}$, with all orders in $\alpha$ accounted

$$
U_{g}=\frac{\partial U_{f}}{\partial \ln (1 / \lambda)},
$$

with $U_{f}=f$ and the Sudakov form factor $f$ is given by Eq.(3).

When we put again $\mu=m$,

$$
g=g^{(1)}(\rho) U_{f}(\rho)=-2 \frac{1}{\rho} \frac{\partial U_{f}}{\partial \ln (\rho)}=-2 \frac{\partial f}{\partial \rho}
$$

where $\rho=1 / \lambda=s / m^{2}$.

Therefore, the double-logarithmic asymptotics of Eq.(1) for the interaction of electron with the external electromagnetic field in the kinematical region (2) is ${ }^{2}$

$$
\Gamma_{\mu}=\left(\gamma_{\mu}+\frac{\sigma_{\mu \nu} q_{\nu}}{m} \frac{\partial}{\partial \rho}\right) \exp \left(-\frac{\alpha}{4 \pi} \ln ^{2} \rho\right)
$$

We have obtained the relation (42) between form factors $f$ and $g$ by direct resummation of the leading contributions of Feynman graphs to all orders in $\alpha$. However, Eq. (43) can be also obtained in much easier way, using the infrared evolution equation (IREE) method proposed in 8 for calculating QCD form factors in DLA. We use this method in Sect. 3 for obtaining form factor $g$ for quark. Below we demonstrate how it works in QED, obtaining again form factor $g$ for electron.

First let us notice that we dropped the electron mass $m$ in denominators of integrals of Eqs. (21)-(28) and replaced it by IR cut-off $\mu$ just for the sake of simplicity of these expressions. It is a consistent procedure in DLA. However, it was not necessary. When both $m$ and $\mu$ are kept ,

$$
g=g^{(1)}\left(s / \mu^{2}\right) U_{f}\left(s / \mu^{2}\right)
$$

instead of Eq. (42). Eqs. (42) and (44) are identical with DL accuracy. However Eq. (44) reads that the form factor $g$ of the electron can be presented as the product of the single-logarithmic first-loop form factor $g^{(1)}$ (which does

\footnotetext{
${ }^{2}$ The exponentiation of the radiative corrections to $g^{(1)}$ in DLA based on was suggested first in work [9] where $g$ was calculated in two loops.
} 
not depend on $\mu$ ) and the $\mu$ - dependent function $U_{f}$ presenting DL contributions of the other, "soft" photons. The factorization

$$
g=g^{(1)}\left(s / m^{2}\right) U\left(s / \mu^{2}\right),
$$

with an unknown function $U\left(s / \mu^{2}\right)$ could be deduced from lower-loop calculations. After that one must calculate $U$ in DLA, which can be done easily using the IREE method. Indeed, although there is no ordering between transverse momenta of virtual photons in DLA, the whole phase space of transverse momenta $k_{i \perp}$ of virtual photons $(i=1, \ldots$. can be regarded as a superposition of the regions, each of them corresponds to a specific ordering between $k_{i \perp}$. Therefore there is always the photon $i_{m}$ with minimal $k_{i_{m} \perp} \equiv k_{\perp}$ (which we call the softest photon) in each of those regions. For such softest photon, the lowest limit of integrating is $\mu$ whereas limits for integrating over other $k_{i \perp}$ do not depend on $\mu$. According to the results of [8] the DL contribution of the softest photon can be factorized in a certain sense. Namely, $k_{\perp}$ acts as a new IR cut-off for integrating over other photon momenta whereas integrating over the longitudinal Sudakov parameters of the softest photon can be done like in the first-loop case, without involving other photon momenta. Therefore the IREE for $g$ is

$$
g^{(1)}\left(s / m^{2}\right) U\left(s / \mu^{2}\right)=g^{(1)}\left(s / m^{2}\right)-g^{(1)}\left(s / m^{2}\right)\left[\left(\frac{\alpha}{2 \pi}\right) \int_{\mu^{2}}^{s} \frac{d k_{\perp}^{2}}{k_{\perp}^{2}} \ln \left(s / k_{\perp}^{2}\right)\right] U\left(s / k_{\perp}^{2}\right)
$$

where the first term in rhs is the first loop contribution and the expression in the square brackets corresponds to the one-loop integration over the softest virtual photon momentum. Differentiating Eq. (46) over $\mu$ leads immediately to

$$
\frac{\partial U}{\partial \ln \left(s / \mu^{2}\right)}=-\frac{\alpha}{2 \pi} \ln \left(s / \mu^{2}\right) U
$$

with obvious solution $U=\exp \left((-\alpha / 4 \pi) \ln ^{2}\left(s / \mu^{2}\right)\right)$ which coincides with Eq. (42).

\section{ELASTIC FORM FACTORS OF THE QUARK}

In the previous section we have shown that the form factor $g$ of the electron can be presented as the product of the single-logarithmic first-loop contribution of a "hard" photon and the independent DL contributions from the other "soft" photons. This factorization property leads to the exponentiation of the one-loop DL contribution. We use below this picture for generalizing (43) to QCD.

The main difference between QCD and QED is that the quark-gluon interaction has an additional colour matrix factor. For the one-loop contribution of Fig. 1 this leads to an additional colour factor $C_{F}$ in Eqs.(18), (66). In the next orders the colour factors of different graphs in Fig. 2 are different: for example in order $\alpha_{s}^{2}$ the colour factors are $C_{F}^{2}$ for the ladder graph and $C_{F}(-1 / 2 N)=C_{F}^{2}-C_{F}(N / 2)$ for the non-ladder one. In the sum, the contributions proportional to $C_{F}^{2}$ combine to reproduce the same result as in $\mathrm{QED}$, with just the replacement of the electromagnetic charge $\alpha$ of the electron by the colour charge $\alpha_{s} C_{F}$ of the quark. The remaining DL contributions which are proportional to $C_{F}(N / 2)$ are cancelled by the QCD graphs with three-gluon vertices. The generalization of this property to many-loop DL contributions for the case of the form factor $f_{q}$ was done in [4]:

$$
f_{q}=\exp \left[-\frac{\alpha_{s} C_{F}}{4 \pi} \ln ^{2} \rho\right] .
$$

The easiest way to prove the exponentiation of DL radiative corrections for the quark form factors $f_{q}$ and $g_{q}$ is to exploit the QCD generalization [8] of the Gribov bremsstrahlung theorem [10] and to use the method of constructing the IREE [8]. According to it, the DL contribution of the virtual photon/gluon with the smallest $k_{\perp}$ can be separated out of the total amplitude as an independent factor and the amplitude can be factorized into the product of this factor and the same amplitude with the new infrared cutoff $k_{\perp}$ instead of $\mu$, see Fig. 3. For $f_{q}$ this approach immediately results in the following IREE:

$$
f_{q}\left(\frac{-q^{2}}{\mu^{2}}\right)=1-\frac{\alpha_{s} C_{F}}{2 \pi} \int_{\mu^{2}}^{-q^{2}} \frac{d k_{\perp}^{2}}{k_{\perp}^{2}} \ln \left(\frac{-q^{2}}{k_{\perp}^{2}}\right) f_{q}\left(\frac{-q^{2}}{k_{\perp}^{2}}\right)
$$

where the unity stands for the Born term and $\ln \left(-q^{2} / k^{2}\right)$ in the integrand comes from integration over the longitudinal components of $k$. Eq. (48) is just the solution to Eq. (49). 
Let us construct the IREE for the form factor $g_{q}$. Motivated by calculations in the lowest orders in $\alpha_{s}$, we assume that

$$
g_{q}=g_{q}^{(1)}\left(\frac{-q^{2}}{m^{2}}\right) U\left(\frac{-q^{2}}{\mu^{2}}\right)
$$

where $g_{q}^{(1)}=-\left(\alpha_{s} C_{F} / \pi\right) \ln \left(-q^{2} / m^{2}\right)$ is the one-loop value for $g_{q}$ and $U$ is an unknown DL function. It is essential that only $U$, not $g_{q}^{(1)}$, depends on the infrared cut-off (recall that we put $\mu=m$ only in the final expressions). Now applying the arguments of ref. [8], reproduced above, in order to construct the IREE for $g_{q}$, we obtain (cf. Eq. (46))

$$
g_{q}^{(1)}\left(\frac{-q^{2}}{m^{2}}\right) U\left(\frac{-q^{2}}{\mu^{2}}\right)=g_{q}^{(1)}\left(\frac{-q^{2}}{m^{2}}\right)-\frac{\alpha_{s} C_{F}}{2 \pi} \int_{\mu^{2}}^{-q^{2}} \frac{d k_{\perp}^{2}}{k_{\perp}^{2}} \ln \left(\frac{-q^{2}}{k_{\perp}^{2}}\right) g_{q}\left(\frac{-q^{2}}{m^{2}}\right) U\left(\frac{-q^{2}}{k_{\perp}^{2}}\right) .
$$

Comparing Eqs. (49) and (51), we conclude that

$$
g_{q}=-2 \frac{\partial f_{q}}{\partial \rho}
$$

just as in QED.

In other words, the whole effect of replacement of the electromagnetic gauge group $U(1)$ by $S U(3)$ is the replacement of $\alpha$ in Eq. (42) by $\alpha_{s} C_{F}$ and we arrive at the following expression for the vertex $\Gamma_{\mu}^{(q)}$ of the quark in an external electromagnetic field in the kinematical region (2):

$$
\Gamma_{\mu}^{(q)}=\left[\gamma_{\mu}+\frac{\sigma_{\mu \nu} q_{\nu}}{m} \frac{\partial}{\partial \rho}\right] \exp \left[-\frac{\alpha_{s} C_{F}}{4 \pi} \ln ^{2} \rho\right]
$$

\section{INELASTIC FORM FACTORS}

The exponential fall of $f$ and $g$ means that non-radiative (elastic) scattering of an electron or quark is suppressed and therefore radiative (inelastic) scattering prevails at large $-q^{2}$. Indeed, in the kinematical region (16) bremsstrahlung processes are known to become essential both in QED and in QCD. In the present section we show that accounting for both gluon and photon bremsstrahlung accounted for in DLA does not change the relation between the form factors we obtained in the previous sections. In the first place let us discuss the case when electron scattering by an external electromagnetic field, considered in section 2, is accompanied by emission of bremsstrahlung photons with momenta $k_{i}(\mathrm{i}=1, . ., \mathrm{n})$. At sufficiently small $k_{i}$, the scattering amplitude for the process is

$$
\Gamma_{\mu}^{(n)}=B_{1} B_{2} \ldots B_{n}\left[\gamma_{\mu} F_{(n)}-\frac{\sigma_{\mu \nu} q_{\nu}}{2 m} G_{(n)}\right]
$$

where the photon bremsstrahlung factors $B_{i}$ are given by Eq. (5), and $F_{(n)}, G_{(n)}$ are the inelastic form factors of the electron. Besides $q^{2}$, these form factors may also depend on the photon momenta $k_{i}$. Again we use the method of ref. [8] to obtain a relation between $F_{(n)}$ and $G_{(n)}$ and therefore we have to introduce the infrared cutoff $\mu$ as a minimal value for the transverse momenta of all the photons involved, both virtual and emitted. After that we must use again the generalization of the Gribov bremsstrahlung theorem see [8] like it was done in previous Sections.

For the sake of simplicity, let us begin with $n=1$ in Eq. (54), considering the emission of a single bremsstrahlung photon. In this case one can represent the scattering amplitude $\Gamma_{\mu}^{(n)}$ by the graphs in Fig. 6 . As in Fig. 3 the blob means that radiative corrections to all orders in $\alpha$ are taken into account in the DLA. As the transverse momenta of the virtual photons are ordered in the DLA, let us denote by $k_{\perp}$ the smallest of them. We have to integrate over $k_{\perp}$. In the DLA the region of integration over $k_{\perp}^{2}$ runs from $\mu^{2}$ to $\sim-q^{2}$. Let us divide it into two subregions: the region where $k_{\perp}$ is the smallest

$$
k_{\perp}<k_{1 \perp}
$$

and the region where $k_{1 \perp}$ is the smallest

$$
k_{\perp}>k_{1 \perp} .
$$


According to the results of [8] the graphs in Fig. 19 a, b correspond to $F_{(1)}$ in the region (56) whereas the graph in Fig. Ac corresponds to $F_{(1)}$ in the region (55). Being on mass-shell (the propagators marked by crosses), all the blobs in Fig. 1 are gauge invariant. The blobs in Fig. 能, b have infrared cutoff $k_{1 \perp}$ and the blob in Fig. 化 has infrared cutoff $k_{\perp}$. Dropping the common factor $B_{1}$ we obtain the following IREE for $F_{(1)}$ :

$$
F_{(1)}\left(q, k_{1}, \mu\right)=f\left(\frac{-q^{2}}{k_{1 \perp}^{2}}\right)-\frac{\alpha}{2 \pi} \int_{\mu^{2}}^{k_{1 \perp}^{2}} \frac{d k_{\perp}^{2}}{k_{\perp}^{2}} \ln \left(\frac{-q^{2}}{k_{\perp}^{2}}\right) F_{(1)}\left(q, k_{1}, k_{\perp}\right) .
$$

Differentiating Eq. (57) with respect to $\mu$ immediately yields that the solution does not depend on $k_{1}$ and coincides with the elastic form factor $f\left(-q^{2} / \mu^{2}\right)$, Eq. (3). This result was obtained earlier in 11] by direct calculation of Feynman graphs in DLA.

As the soft bremsstrahlung of the photon $k_{1}$ does not influence the spinor structure of the blobs in Fig. 团, we can easily generalize the IREE (57) to the case of the form factor $G_{(1)}$, just substituting it for $F_{(1)}$ and $g$ for $f$. Due to the infrared stability of the one-loop result $g^{(1)}$ one can consider it to be a common factor independent of $\mu$.

Using

the ansatz

$$
G_{(1)}=g^{(1)}\left(s / m^{2}\right) U_{(1)}\left(s / \mu^{2}\right),
$$

we immediately obtain $U_{(1)}=F_{(1)}$. Repeating the same method for $n=2,3, .$. emitted photons, we conclude that

$$
G_{(n)}=-2 \frac{\partial F_{(n)}}{\partial \rho} .
$$

The fact that neither $F_{(n)}$ nor $G_{(n)}$ depends on the bremsstrahlung photon momenta implies factorization of the bremsstrahlung and a Poisson energy spectrum for the soft photons.

Now let us turn to QCD and consider the process where instead of the electron a quark interacts with an external electromagnetic field, and take into account emission of $n$ bremsstrahlung gluons. As each emitted gluon may emit other gluons, producing gluon cascades, the gluon bremsstrahlung factors and the whole picture are more complicated. As was shown in [7], in DLA all the bremsstrahlung gluons inside a cascade, or jet, are strongly ordered in energies and emission angles, relative to their parent gluons. This is a result of destructive interference or the coherence effect. Therefore one has to use different representations for the scattering amplitude $\tilde{\Gamma}_{\mu}^{(n)}$ for each DL kinematical region, accounting for the various ways to group $n$ gluons into jets. In each such region one can expect that the scattering amplitude $\tilde{\Gamma}_{\mu}^{(n)}$ takes the simple form of a product of the gluon bremsstrahlung factors $\tilde{B}_{i}$ and the inelastic form factors $\tilde{F}_{(n)}$ and $\tilde{G}_{(n)}$

$$
\tilde{\Gamma}_{\mu}^{(n)}=\tilde{B}_{1} \ldots \tilde{B}_{n}\left[\gamma_{\mu} \tilde{F}_{(n)}-\frac{\sigma_{\mu \nu} q_{\nu}}{2 m} \tilde{G}_{(n)}\right] .
$$

To make the problem clearer let us consider the simplest case $n=1$. Then besides the graphs of Fig. 1 the two new graphs of Fig. . 5 give DL contributions to $\tilde{F}_{(1)}$ and the IREE (57) takes the following form:

$$
\tilde{F}_{(1)}\left(q, k_{1} ; \mu\right)=f\left(\frac{-q^{2}}{k_{1 \perp}^{2}}\right)-\frac{\alpha_{s}}{2 \pi} \int_{\mu^{2}}^{k_{1 \perp}^{2}} \frac{d k_{t}^{2}}{k_{t}^{2}}\left[C_{F} \ln \left(\frac{-q^{2}}{k_{t}^{2}}\right)+\frac{N}{2} \ln \left(\frac{2\left(p_{1} k_{1}\right)}{k_{t}^{2}}\right)+\frac{N}{2} \ln \left(\frac{2\left(p_{2} k_{1}\right)}{k_{t}^{2}}\right)\right] \tilde{F}_{(1)}\left(q, k_{1} ; k_{t}\right)
$$

where we have accounted for the different colour factors for the graphs in Fig. Ac and in Fig. 5. It is worthwhile to mention that in each term of the integrand in Eq. (61) $k_{t}$ must be defined separately, with respect to the momenta $p_{1}$ and $p_{2}$ for the first term, $p_{1}$ and $k_{1}$ for the second term and $p_{2}, k_{1}$ for the last term (see [8] for details).

Due to the kinematical relation

$$
2\left(p_{1} k_{1}\right) 2\left(p_{2} k_{1}\right)=\left(-q^{2}\right) k_{1 \perp}^{2}
$$

we obtain the following solution to Eq. (61)

$$
\tilde{F}_{(1)}=\exp \left(-\frac{\alpha_{s}}{4 \pi}\left[C_{F} \ln ^{2}\left(\frac{-q^{2}}{\mu^{2}}\right)+\frac{N}{2} \ln ^{2}\left(\frac{k_{1 \perp}^{2}}{\mu^{2}}\right)\right]\right) .
$$

In contrast to the inelastic form factor of the electron, Eq.(3), the form factor of the quark $\tilde{F}_{(1)}$ depends on $k_{1}$. This implies the violation of both factorization and the Poisson energy spectrum for gluon bremsstrahlung. 
The expression obtained for $\tilde{F}_{(1)}$ is the exponentiation of the one-loop contribution. The same exponentiation was shown in [8] to be true for the general case of $n$ bremsstrahlung gluons, $\tilde{F}_{(n)}$.

Obviously, if we keep the ansatz

$$
G_{(n)}=g^{(1)}\left(q, k_{1}, m\right) \tilde{U}_{(n)}\left(q, k_{1}, \mu\right)
$$

the above argument holds for constructing the IREE of the DL function $\tilde{U}_{(n)}\left(q, k_{1}, \ldots, k_{n} ; \mu\right)$ and for it being equal to $\tilde{F}_{(n)}\left(q, k_{1}, \ldots, k_{n} ; \mu\right)$, which results in the same relation, Eq. (59).

\section{CONCLUSION}

We have calculated the electromagnetic form factors of the electron and quark in the asymptotic regime where the momentum $q$ transferred to the electron or quark is much greater than their masses. The Eqs. (43) and (53) that we have obtained predict an exponential fall for the form factors when $q^{2}$ increases. This corresponds to the suppression of non-radiative scattering, without photon bremsstrahlung, at high energies. When photon bremsstrahlung is taken into account in the DLA, in the expressions for the radiative (inelastic) form factors Eqs. (43) and (53) are multiplied by the bremsstrahlung factors $B_{i}$ defined in Eq. (5). All the form factors in Eqs. (43) and (53) are independent of momenta of the emitted photons. This means that taking into account the form factor $g$ does not violate the Poisson energy spectrum for the photon bremsstrahlung in the double logarithmic approximation. In contrast, the inelastic form factor that accounts for gluon bremsstrahlung depends, in the DLA, both on the emitted gluon momenta and on the structure of each of the gluon cascades [7.8]. This makes it possible to obtain simple expressions for the inelastic form factor in QCD only separately for every kinematical region corresponding to a certain ordering of the emission energies and angles [7]. Still, in the DLA, even with that complication, the relation Eq. (59) holds also for the two quark form factors.

\section{ACKNOWLEDGEMENTS}

We are grateful to G. Altarelli, M. Mangano, N. Nikolaev and D. Soper for useful discussions. The work was supported in part by the grant INTAS-97-30494.

[1] J.Schwinger. Phys. Rev. 73 (1948) 416.

[2] A.Czarnecki and W.J.Marciano. BNL-HET-98/43; hep-ph/9810512.

[3] V.V.Sudakov. ZhETP 3 (1956) 65.

[4] J.J.Carazzone, E.C.Poggio and H.R.Quinn. Phys. Rev. D11 (1975) 2286; J.M.Cornwall and G.Tiktopoulos. Phys. Rev. D13 (1976) 3370.

[5] V.G.Gorshkov. ZhETP 56 (1969) 598.

[6] V.S.Fadin and E.A.Kuraev. Sov. J. Nucl. Phys. 27 (1983) 587.

[7] B.I.Ermolaev and V.S.Fadin.JETP Lett. 33 (1981) 269; V.S.Fadin. Sov.J.Nucl.Phys. 37 (1983) 2145.

[8] B.I.Ermolaev, V.S.Fadin and L.N.Lipatov. Sov. J. Nucl. Phys. 45 (1987) 508.

[9] R.Barbieri, J.A.Mignaco and E.Remiddi. Nuovo Cimento 11A (1972) 824.

[10] V.N.Gribov. Sov. J. Nucl. Phys. 5 (1967) 280.

[11] V.G.Gorshkov. ZhETP 56 (1969) 597. 


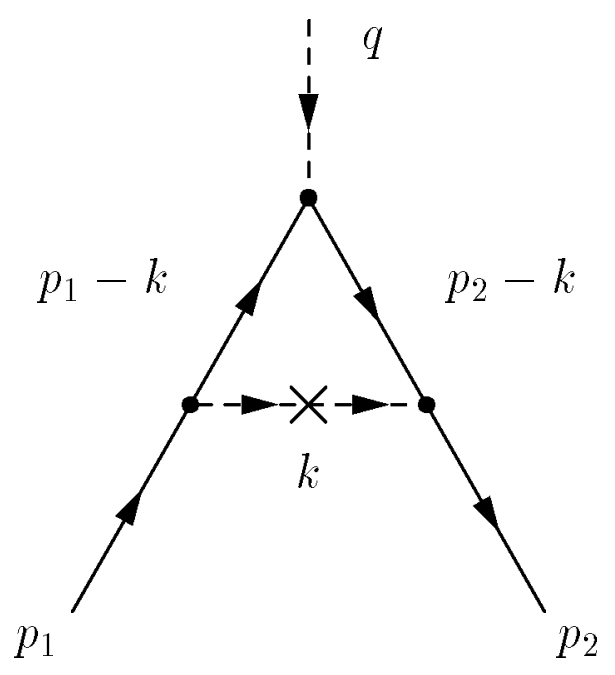

FIG. 1. One-loop diagram for $\Gamma_{\mu}$.

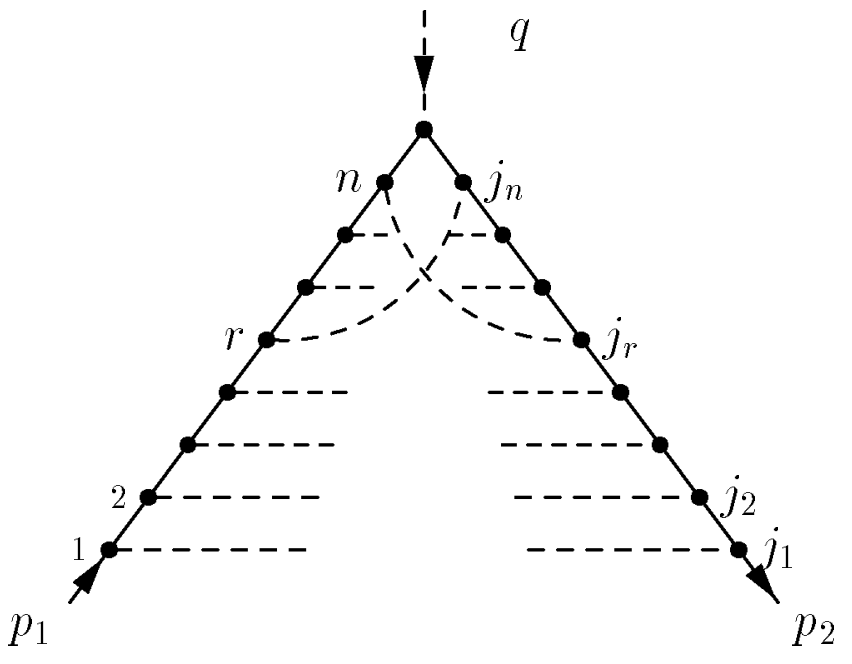

FIG. 2. General diagram for $\Gamma_{\mu}$ in DLA.
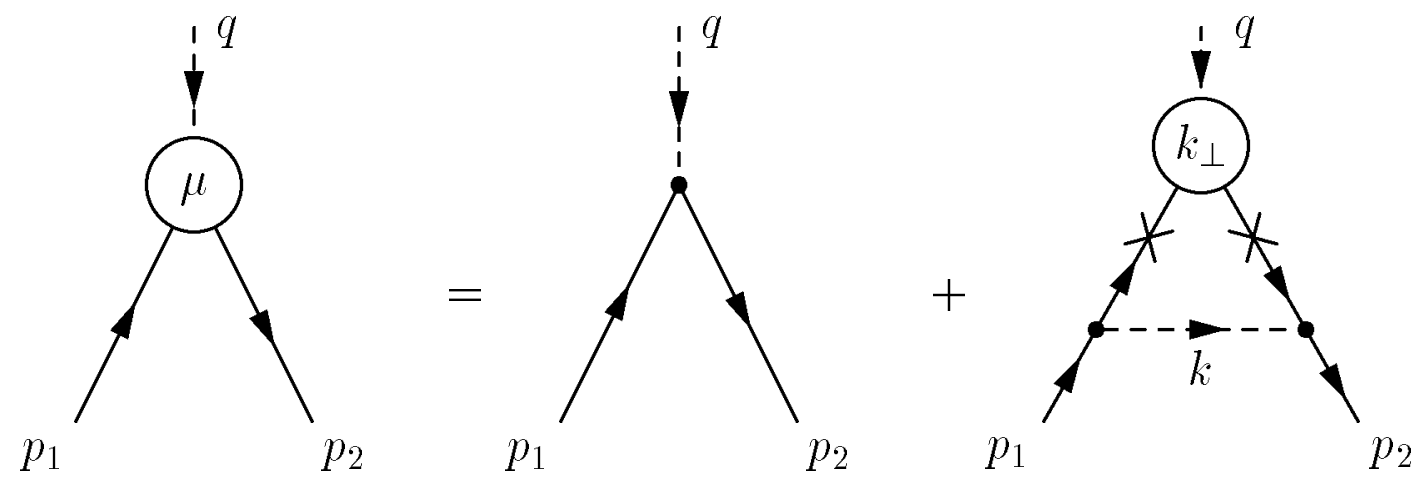

FIG. 3. The IREE equation for the elastic form factor $f$ of electron and quark. 


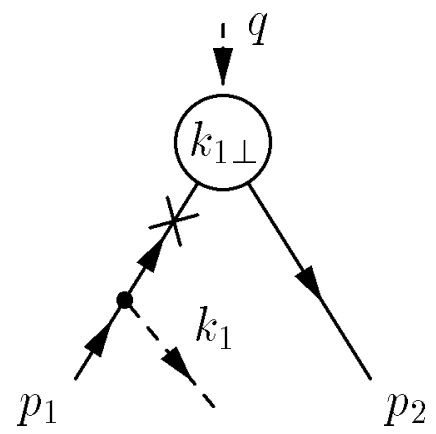

(a)

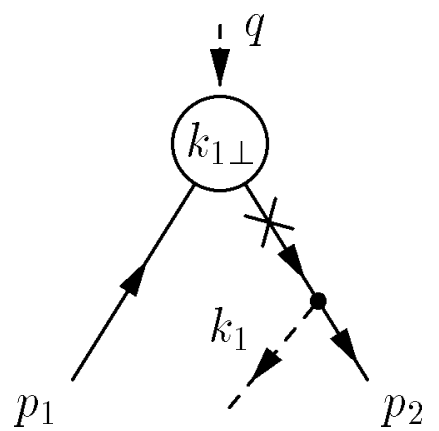

(b)

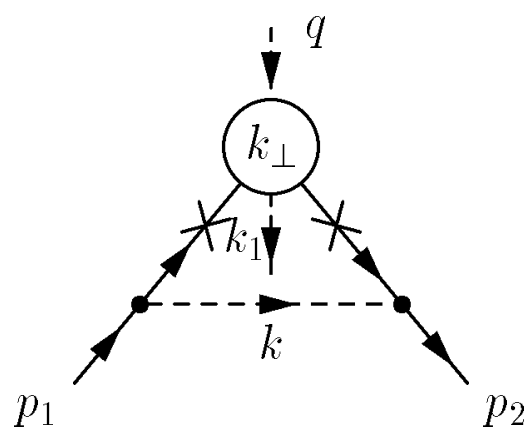

(c)

FIG. 4. Graphs for inelastic form factors of electron.

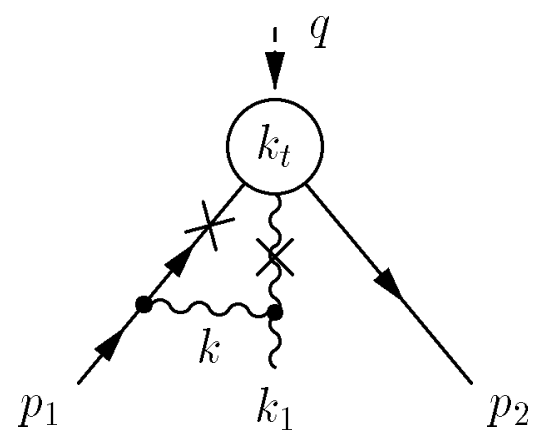

(a)

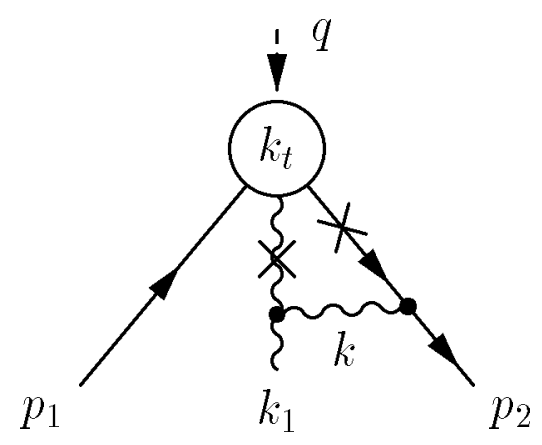

(b)

FIG. 5. 3-gluon graphs for inelastic form factors of quark. 